\title{
Enhancing solubility of deoxyxylulose phosphate pathway enzymes for microbial isoprenoid production
}

\author{
Kang Zhou', Ruiyang Zou', Gregory Stephanopoulos ${ }^{1,3}$ and Heng-Phon Too ${ }^{1,2^{*}}$
}

\begin{abstract}
Background: Recombinant proteins are routinely overexpressed in metabolic engineering. It is well known that some over-expressed heterologous recombinant enzymes are insoluble with little or no enzymatic activity. This study examined the solubility of over-expressed homologous enzymes of the deoxyxylulose phosphate pathway (DXP) and the impact of inclusion body formation on metabolic engineering of microbes.

Results: Four enzymes of this pathway (DXS, ISPG, ISPH and ISPA), but not all, were highly insoluble, regardless of the expression systems used. Insoluble dxs (the committed enzyme of DXP pathway) was found to be inactive. Expressions of fusion tags did not significantly improve the solubility of dxs. However, hypertonic media containing sorbitol, an osmolyte, successfully doubled the solubility of dxs, with the concomitant improvement in microbial production of the metabolite, DXP. Similarly, sorbitol significantly improved the production of soluble and functional ERG12, the committed enzyme in the mevalonate pathway.

Conclusion: This study demonstrated the unanticipated findings that some over-expressed homologous enzymes of the DXP pathway were highly insoluble, forming inclusion bodies, which affected metabolite formation. Sorbitol was found to increase both the solubility and function of some of these over-expressed enzymes, a strategy to increase the production of secondary metabolites.
\end{abstract}

Keywords: Isoprenoids, Protein solubility, Deoxyxylulose phosphate pathway, Activity analysis, Metabolic engineering

\section{Introduction}

Isoprenoids, a large family of natural compounds including many plant based pharmaceuticals such as artemisinin [1] and paclitaxel [2], are produced by the deoxyxylulose phosphate (DXP) pathway and/or the mevalonate (MVA) pathway in nature [3]. The current industrial isoprenoid production methods include direct extraction from plants and semi-synthesis using plant metabolites [4]. These processes are all restricted by the supply of specific plant materials, which are often affected by unpredictable factors including variations in weather and market fluctuations [5].

\footnotetext{
* Correspondence: heng-phon_too@nuhs.edu.sg

${ }^{1}$ Chemical and Pharmaceutical Engineering, Singapore-MIT Alliance, 4 Engineering Drive 3, Singapore, Singapore

${ }^{2}$ Department of Biochemistry Yong Loo Lin School of Medicine, National University of Singapore, 8 Medical Drive Blk MD7 \#05-04, Singapore 117597, Singapore

Full list of author information is available at the end of the article
}

To improve sustainability and production capacity of isoprenoids, heterologous biosynthesis from economical carbon sources in microbes has been intensively studied in the past decade $[2,5]$. To date, there have been many successful reports of using the DXP pathway to produce isoprenoid nutraceuticals (e.g. lycopene [6-10] and other carotenoids $[11,12])$ and pharmaceuticals (e.g. artemisinin precursors [13,14] and paclitaxel precursors [2]).

To increase carbon flux through the DXP pathway, the enzymes involved were overexpressed [2,6-14] to catalyze the bio-transformations of the DXP metabolites in vivo. So far, four enzymes (DXS, IDI, ISPD and ISPF) have been identified to be rate limiting based on a series of empirical studies $[9,15]$. The expression levels of these four enzymes have also been semi-empirically optimized for paclitaxel precursor production [2]. It is well accepted that some recombinant proteins can form insoluble aggregates (termed as inclusion bodies), generally regarded to be functionally 
inactive [16]. However, the extents to which the overexpressed recombinant endogenous DXP enzymes forming inclusion bodies and their impacts on the flux through the pathway have yet to be systematically investigated.

This study examined the solubility status of all the DXP enzymes when overexpressed and attempted to demonstrate the importance of protein solubility in the production of secondary metabolites. Computational prediction was initially explored to evaluate the solubility status and empirical verifications were carried out in E. coli. An unanticipated and critical observation is that many DXP enzymes (DXS, ISPA, ISPG and ISPH) were found to be highly insoluble. Interestingly, the enzymes IDI, ISPD and ISPF, thought to be rate-limiting and hence useful for the enhancement of isoprenoids production [9], were found to be highly soluble. From these observations, it is now necessary to reevaluate the use of the other highly insoluble DXP enzymes for enhancing isoprenoid production. Attempts were also made to optimize the solubility of the insoluble enzymes and to examine the enhancements in isoprenoid production.

\section{Results}

Solubility of over-expressed recombinant DXP

\section{pathway enzymes}

DXP pathway has so far been characterized to be a linear pathway [3], producing isopentenyl diphosphate (IPP) and dimethylallyl diphosphate (DMAPP) from pyruvate and glyceraldehyde 3-phosphate (GAP), two important metabolites in central metabolism (Figure 1). IPP and DMAPP (C5) are further assembled into geranyl diphosphate (GPP, C10) and farnesyl diphosphate (FPP, C15), precursors for all C10 and C15 isoprenoids [4]. To date, little is known of the solubility of the enzymes involved in this pathway when overexpressed for the production of isoprenoids.

As a first attempt, the solubility of the enzymes in the DXP pathway was evaluated by in silico modeling. Several correlation algorithms built on published experimental data have been reported [17-21]. Revised WH method [18], one of the most commonly used and accurate methods [21], was used to predict solubility of the DXP pathway enzymes. Some of the enzymes (DXS, ISPE and ISPG) were predicted by these methods to be insoluble when overexpressed in E. coli (Table 1). Similarly, in vitro expression study [22] showed that a subgroup of the DXP pathway proteins (DXS and IDI) were insoluble (Table 1). As in silico prediction did not completely agree with the published in vitro expression data, it was essential to determine the solubility of the enzymes when overexpressed in vivo.

To verify that some of the DXP enzymes when overexpressed were differentially soluble, each of the enzymes was expressed individually in three distinct expression systems in different strains of E. coli (BL21 strain - T7 promoter,

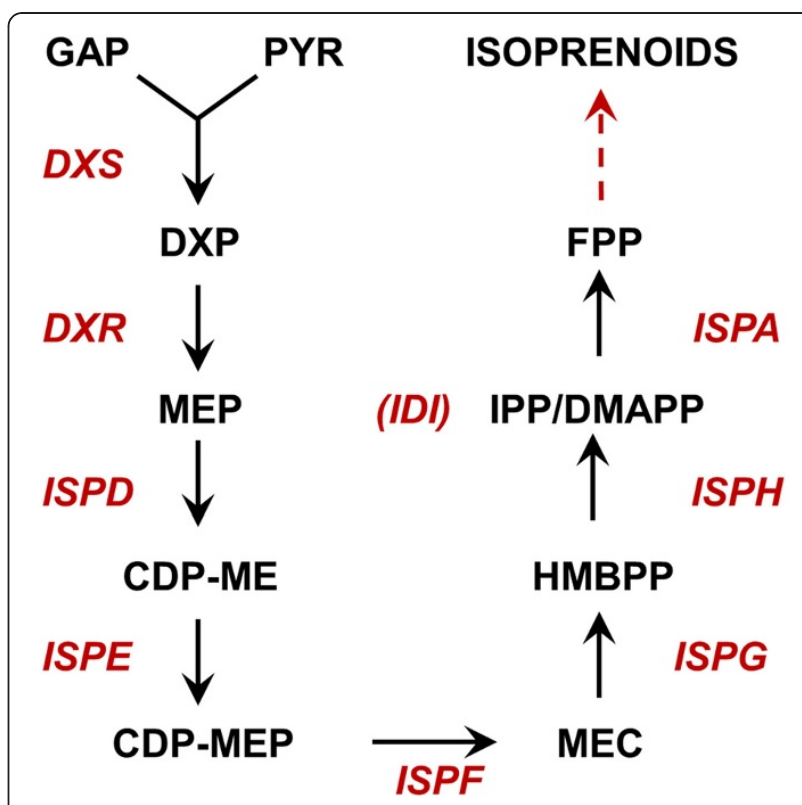

Figure 1 Metabolites and enzymes related to the DXP pathway. GAP: glyceraldehyde-3-phosphate; PYR: pyruvate; DXP: 1-deoxy-Dxylulose 5-phosphate; MEP: 2C-methyl-D-erythritol 4-phosphate; CDP-ME: 4-diphosphocytidyl-2C-methyl D-erythritol; CDP-MEP: 4diphosphocytidyl-2C-methyl D-erythritol 2-phosphate; MEC: 2Cmethyl-D-erythritol 2,4-diphosphate; HMBPP: (E)-4-hydroxy-3-methylbut-2-enyl pyrophosphate; IPP: Isopentenyl diphosphate; DMAPP: Dimethylallyl diphosphate; FPP: Farnesyl diphosphate; DXS: 1-deoxyD-xylulose 5-phosphate synthase; DXR: 1-deoxy-D-xylulose 5phosphate reductase; ISPD: 4-diphosphocytidyl-2C-methyl-Derythritol synthase; ISPE: 4-diphosphocytidyl-2-C-methylerythritol kinase; ISPF: 2-C-methyl-D-erythritol 2,4-cyclodiphosphate synthase; ISPG: 1-hydroxy-2-methyl-2-(E)-butenyl 4-diphosphate synthase; ISPH: 1-hydroxy-2-methyl-2-(E)-butenyl 4-diphosphate reductase; IDI: isopentenyl diphosphate isomerase; ispA: farnesyl diphosphate synthase.

M15 strain - T5 promoter and DH10B strain - araBAD promoter) at two temperatures $\left(37^{\circ} \mathrm{C}\right.$ and $\left.20^{\circ} \mathrm{C}\right)$. The standard dosage of inducers were used to trigger expression of the proteins (10mM L-arabinose or 1mM IPTG $[6,23])$. In

Table 1 Solubility of the enzymes in the DXP pathway predicted in silico and determined in vitro

\begin{tabular}{lcc}
\hline Protein & $\begin{array}{c}\text { In silico predicted } \\
\text { solubility [18] }\end{array}$ & $\begin{array}{c}\text { In vitro determined } \\
\text { solubility [22] }\end{array}$ \\
\hline DXS & insoluble & $22 \%$ \\
DXR & soluble & N.D.* \\
ISPD & soluble & $67 \%$ \\
ISPE & insoluble & $100 \%$ \\
ISPF & soluble & $84 \%$ \\
ISPG & insoluble & N.D. \\
ISPH & soluble & $75 \%$ \\
IDI & soluble & $32 \%$ \\
ISPA & soluble & $85 \%$ \\
\hline
\end{tabular}

*N.D.: not detected. 
general, solubility of the proteins varied significantly (5\% to $90 \%$, Figure 2). The large variances in solubility across proteins suggested that the method for identifying and quantifying protein solubility is unbiased. This protein solubility analysis method (similar to that used in [21]) was also further validated by filtration studies (Additional file 1). A group of the DXP enzymes (DXS, ISPG, ISPH and ISPA) were indeed found to be highly insoluble (solubility less than $30 \%$ in all conditions examined) (Figure 2). DXS (the committed enzyme of DXP pathway) was previously identified to be crucial (rate-limiting) for isoprenoid production, was found to be highly insoluble in the present study. Using $\mathrm{dxs}$ as a prototype of highly insoluble enzymes, we next examined the impact of inclusion body formation on metabolic engineering of $E$. coli for isoprenoid production.

\section{Enzymatic activity of insoluble recombinant DXS}

Although some inclusion bodies formed with certain enzymes were reported to be active [24], it is generally accepted that inclusion bodies contain primarily incorrect folded proteins and are functionally inactive [25]. To test whether insoluble DXS is catalytically functional, lysates containing recombinant insoluble DXS was characterized by an in vitro assay, where DXS activity was determined by measuring the formation of DXP. It was found that
DXP was produced at low levels (less than $1 \mu \mathrm{M}$ ) with insoluble DXS containing lysates. As a comparison, the same amount of purified soluble DXS (Figure $3 \mathrm{~B}$ ) was spiked into the lysates, and high level of DXP $(\sim 700 \mu \mathrm{M})$ was produced (Figure $3 \mathrm{~A}$ ), confirming that specific activity of insoluble DXS was significantly lower than that of soluble DXS. This observation suggested that strategies to increase the solubility of DXS may confer higher activity and metabolic flux for isoprenoid production in vivo.

\section{Improving solubility of DXS enhanced the production of DXP}

Improvement of recombinant protein solubility has been intensively studied for the purpose of overproducing soluble proteins, and various effective strategies have been reported, such as lowering incubation temperature [25], use of fusion partner [26], overexpression of chaperone proteins [27] and protein mutagenesis [28]. Recently, Prasad et al. reported a simple yet effective approach to increase the solubility of recombinant proteins, where sorbitol at high concentration reduced protein aggregation in E. coli [29]. To test if this approach could increase the solubility of DXS, high concentration of sorbitol was added directly to the cells in culture. The solubility of DXS was examined and found to be significantly increased (Figure $4 \mathrm{~A}$ and $\mathrm{B}$ ). Other
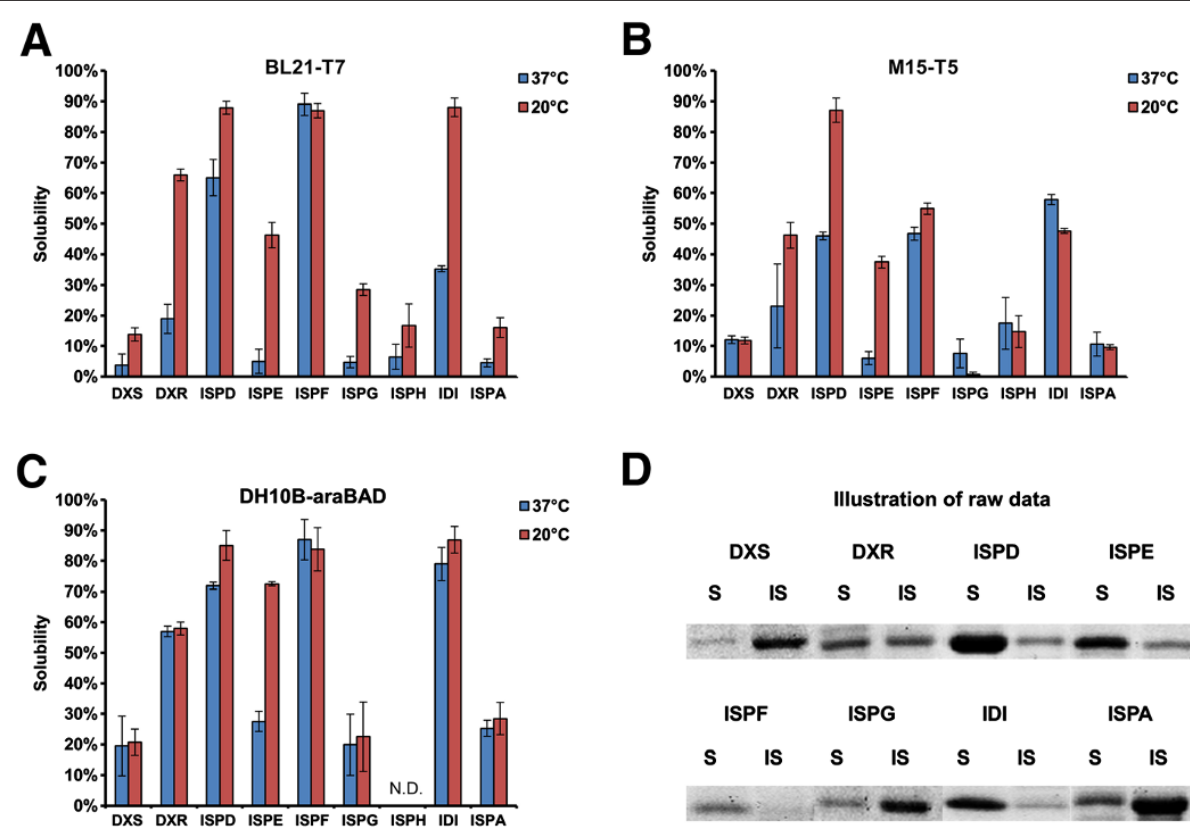

D

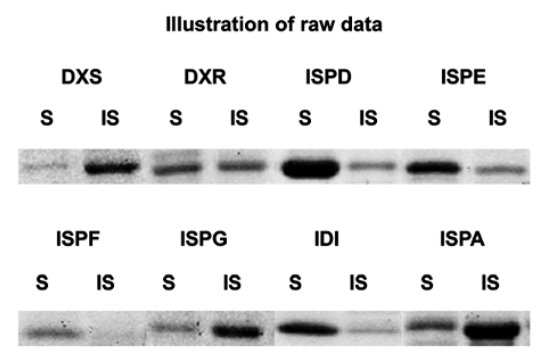

Figure 2 Solubility of DXP enzymes in E. coli. The expression systems and incubation temperatures played important roles in production of soluble proteins. All the enzymes in the DXP pathway were individually expressed by three expression systems (A: BL21 strain - T7 promoter; B M15 strain - T5 promoter; $\mathrm{C}$ : DH10B strain - araBAD promoter) at two temperatures $\left(37^{\circ} \mathrm{C}\right.$ and $\left.20^{\circ} \mathrm{C}\right)$. Presented data were average of triplicates and standard errors were drawn on the plot. The quantifications were based on image of SDS-PAGE gels, some of which (the set for DH10B strain - araBAD promoter, $20^{\circ} \mathrm{C}$ ) were demonstrated in $\mathbf{D}$. S: soluble protein fraction; IS: insoluble protein fraction. The pictures of DXP proteins were cropped from individual gel images and aligned for demonstration purpose. The protein solubilities were generally higher in $20^{\circ} \mathrm{C}$ than those in $37^{\circ} \mathrm{C}$ in all systems (Figure $2 \mathrm{~A}-\mathrm{C}$ ). 


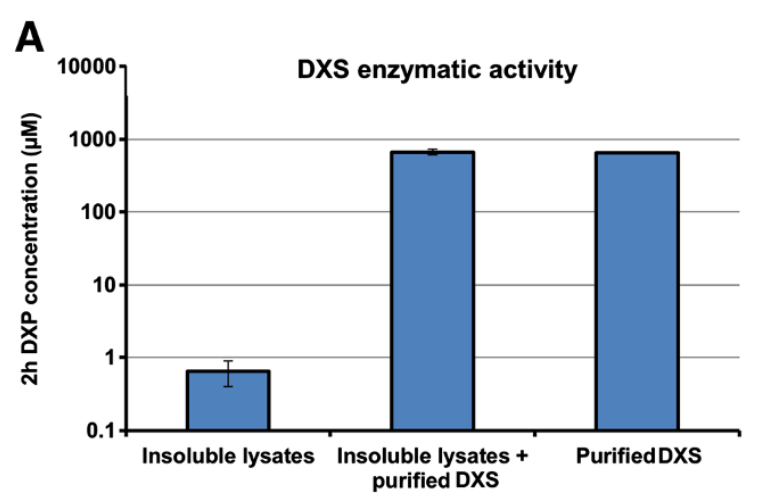

B Protein quantification

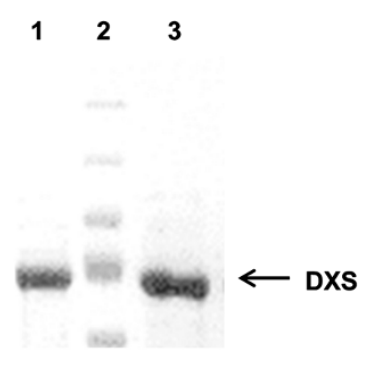

Figure 3 In vitro activity analysis of insoluble DXS. Activity of insoluble DXS was found to be much lower than that of equal amount of soluble DXS by quantifying the quantity of DXP they produced in vitro. A: Quantification of the in vitro produced DXP by insoluble DXS, insoluble DXS + purified DXS (soluble) and purified DXS (soluble), presented data were average of triplicates and standard errors were drawn on the plot; B: Quantification of the insoluble DXS and the purified DXS (soluble) used in A, lane 1: the cell lysate containing insoluble DXS, lane 2: protein markers, lane 3: purified soluble DXS, the arrowed band was DXS.

chemicals, including osmolytes (betaine [30]) and buffering agents (HEPES, phosphate) did not improve the solubility of dxs significantly (Figure $4 \mathrm{~A}$ ). In addition, sorbitol similarly improved the solubility of some but not all other DXP enzymes (Additional file 2).
To demonstrate that improved solubility of DXS results in enhanced production of DXP (committed metabolic intermediate in the DXP pathway), cells grown in sorbitol were lysed and the extracts quantified by LC-MS. It was found that concentrations of DXP were significantly
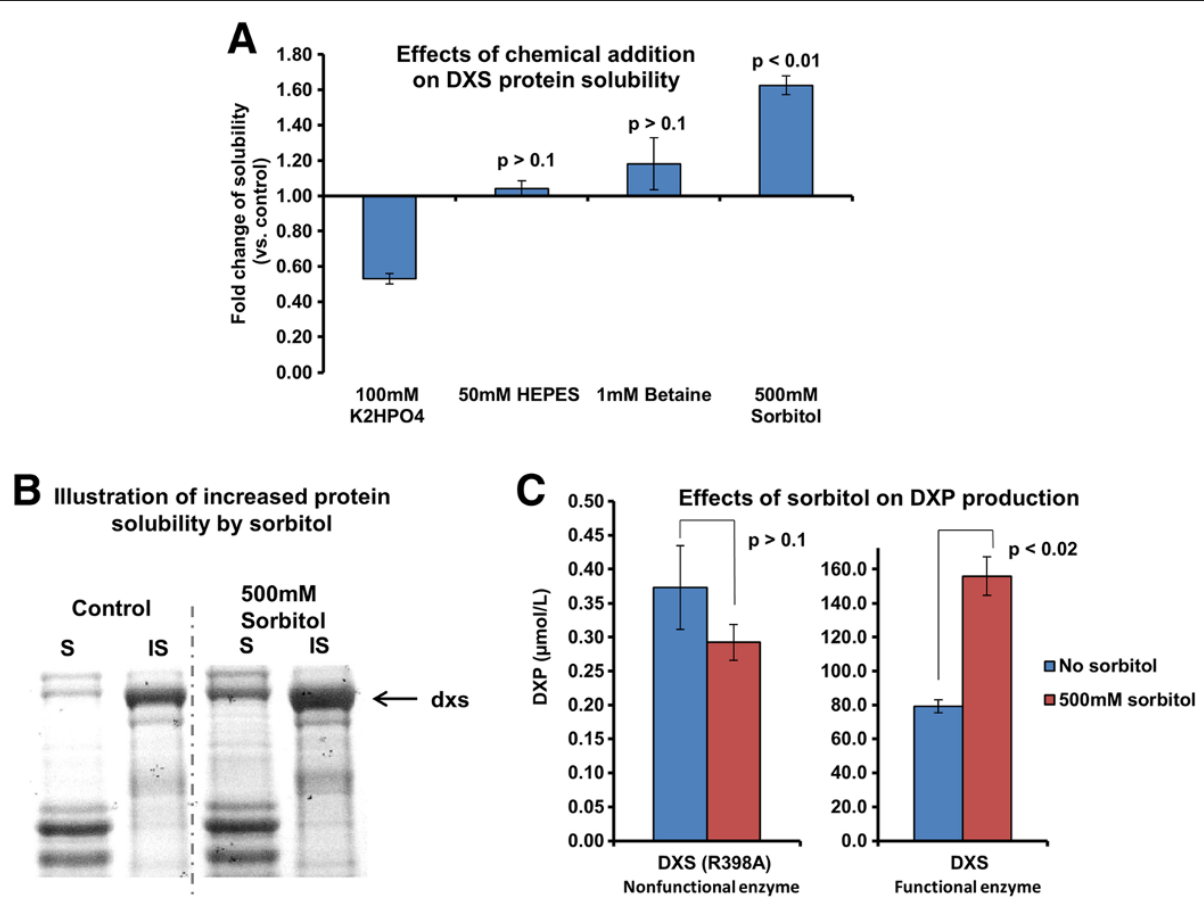

Figure 4 Addition of chemicals for increasing solubility of DXS and production of deoxyxylulose phosphate (DXP). Chemicals with different properties were used to increase solubility of $\mathrm{dxs}$. The expression system (araBAD promoter-DH10B strain) and the temperature $\left(20^{\circ} \mathrm{C}\right.$ ) which produced the most soluble DXS were used here. The use of high concentration of sorbitol was found to significantly increase solubility of DXS and production of DXP (product of DXS). A: Effects of the chemical additions on solubility of DXS; $\mathbf{B}$ : Illustration of increased protein solubility by sorbitol, S: soluble fraction, IS: insoluble fraction; the arrowed band was DXS; C: Effects of sorbitol addition on production of DXP; addition of sorbitol increased DXP production in the cells expressing DXS but not in the ones expressing DXS (R398A), a nonfunctional mutant. Presented data were average of triplicates and standard errors were drawn on the plot. Student's t-test was used to calculate the $p$ values in the statistical analysis. 
higher in sorbitol treated cells as compared to control cells (Figure $4 \mathrm{C}$ ). Addition of sorbitol did not alter the production of DXP with cells overexpressing a nonfunctional DXS (Figure $4 \mathrm{C}$, the construction and characterization of the nonfunctional dxs was described in Additional file 3), indicative that the effect of sorbitol on cells overexpressing functional enzyme was likely be due to the increase in the concentration of soluble DXS. A parallel increase in the concentrations of MEP and MEC (Figure 1) were also observed (Additional file 4), suggesting that sorbitol increased the flux through the entire DXP pathway in cells overexpressing DXS.

\section{Improvement of ERG12 solubility and overproduction of mevalonate phosphate}

To extend the observation of the effect of sorbitol, a critical enzyme (ERG12) in the mevalonate pathway (the other isoprenoid precursor producing pathway, Figure 5 A) was investigated. More than half of overexpressed ERG12 was insoluble and sorbitol was similarly found to enhance the solubility of this enzyme (Figure $5 \mathrm{~B}$ ). In line with the hypothesis that increased solubility confers higher enzymatic activity and better productivity of the respective metabolite, the production of mevalonate phosphate (MVAP) was doubled in the presence of high concentrations of sorbitol (Figure $5 \mathrm{C}$ ). Since the MVA pathway is not endogenous to E. coli (Figure $5 \mathrm{~A}$ ), the production and accumulation of MVAP was attributed to the enzymatic activity of ERG12.

\section{Discussion}

This study addressed an important and often overlooked issue of the solubility of over-expressed recombinant homologous or heterologous enzymes in metabolic engineering. Specifically, we investigated the solubility status of overexpressed DXP enzymes and a heterologous enzyme of the MVA pathway and the impacts on the production of critical precursor metabolites (DXP or MVAP), which are building blocks of all the isoprenoids. It was unexpected
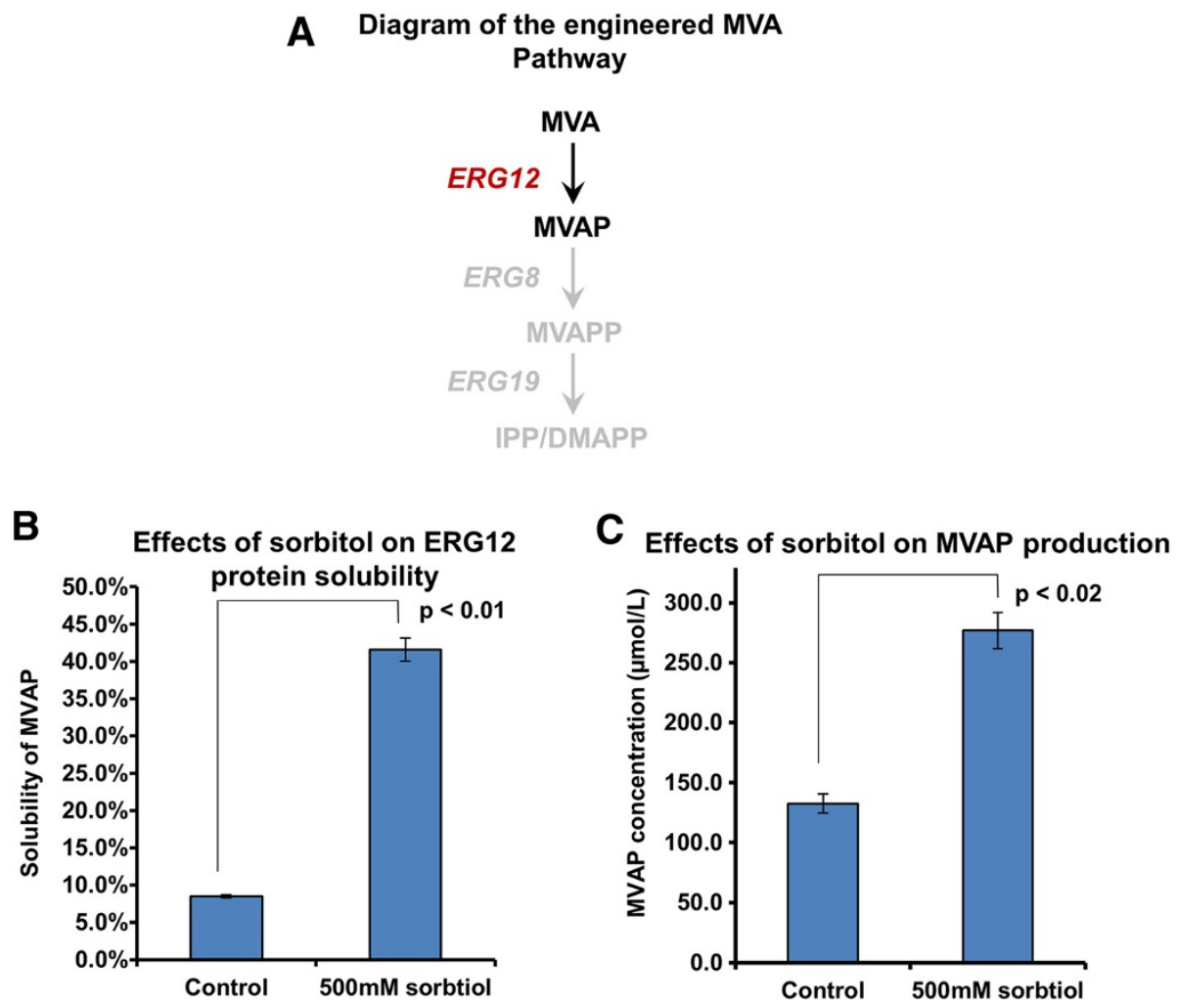

Figure 5 Addition of chemicals for increasing solubility of ERG12 and production of mevalonate phosphate (MVAP). The use of high concentration of sorbitol was also found to increase solubility of ERG12, a critical enzyme in the mevalonate pathway (the other isoprenoid precursor production pathway), and production of MVAP (product of ERG12). A: Metabolites and enzymes related to the mevalonate (MVA) pathway; MVA: Mevalonate, MVAP: Mevalonate phosphate, MVAPP: Mevalonate diphosphate, IPP: Isopentenyl diphosphate, DMAPP: Dimethylallyl diphosphate; ERG12: mevalonate kinase, ERG8: mevalonate phosphate kinase, ERG19: mevalonate diphosphate decarboxylase; B: Effects of sorbitol addition on solubility of ERG12, S: soluble fraction, IS: insoluble fraction; C: Effects of sorbitol addition on production of MVAP; Since ERG8 and ERG19 were not present in E. coli and also not recombinantly expressed, MVAP accumulated in ERG12 expressing E. coli, directly indicating activity of ERG12. Presented data were average of triplicates and standard errors were drawn on the plot. Student's t-test was used to calculate the $p$ values in the statistical analysis. 
that four out of nine enzymes in DXP pathway (DXS, ISPA, ISPG and ISPH) were highly insoluble, despite being endogenous enzymes. Overexpression of DXS resulted in the accumulation of highly insoluble and non-functional $(<1 \%$ activity of the equivalent soluble form) enzyme. This observation cautions against the assumption that overexpression of an enzyme necessarily confers higher enzymatic activity. Interestingly, the combinatorial screening study [9] based on this contentious assumption identified three ratelimiting DXP enzymes (IDI, ISPD and ISPF), which incidentally were found to be highly soluble (Figure 2). It is thus not unreasonable to speculate that the previously thought to be 'non rate-limiting' enzymes found to be insoluble in this study, may serve to enhance the productions of isoprenoids when expressed in soluble forms.

Using DXS as a model enzyme, four commonly used fusion partners, trxA [31], nusA [18], slyD [32] and malE [33] were fused at the N-terminus of DXS in the attempt to increase solubility. The use of these fusion partners did not significantly increase the solubility of DXS (Additional file 5). The effectiveness of the fusion partners in enhancing protein solubility is largely protein-dependent and unpredictable [26,34]. Cysteine residues on surface of DXS (C32, C330 and C457), may form non-specific disulfide bonds and result in protein aggregation [28]. Site-directed mutagenesis of these residues also did not improve solubility (Additional file 6), suggesting that the aggregation of dxs protein was not due to disulfide bond mediated interactions.

Osmolytes have been shown to improve overexpressed proteins in E. coli [29]. Sorbitol at high concentrations significantly improved DXS solubility and the production of the metabolic product (DXP) in E. coli, indicating that solubility of recombinant enzymes is an important factor in the production of secondary metabolites. Consistent with this suggestion was that ERG12, another model enzyme, also showed improved solubility and secondary metabolite production in the presence of sorbitol. The reason why the metabolic intermediates (DXP, MEP and MEC etc.) instead of final product (lycopene etc.) were used as read-out for characterization of DXS was that rate limiting step (ISPG) existed between the intermediates and the isoprenoid products (Zhou et al. PLoS One, In Press, Additional file 7). It is worthy to note that the use of sorbitol is acceptable as a proof-of-concept but may not be routinely used in microbial fermentation due simply to the cost involved [29]. An alternative is to modify the host microbes (such as manipulation of cellular protein folding system $[27,35])$ to render these proteins more soluble for industrial applications.

\section{Conclusion}

In this study, about half of the nine DXP proteins (DXS, ISPG, ISPH and ISPA) were found to be highly insoluble when overexpressed in E. coli. Insoluble DXS, the committed enzyme of the DXP pathway, showed significantly less enzymatic activity when compared to the equivalent amount of soluble enzyme in vitro. High concentration of sorbitol successfully increased the solubility of DXS and resulted in a parallel increase in the metabolic product (DXP). The strategy also improved both solubility and secondary metabolite production of ERG12, a critical enzyme in the mevalonate pathway. This study highlighted the importance of protein solubility in metabolic engineering of microbes for the overproduction of isoprenoids.

\section{Methods}

\section{Bacteria strains and plasmids}

Bacteria strains and plasmids used in this study were summarized in Additional file 8. All the DXP genes were amplified from E. coli genomic DNA and cloned into the modified pBAD-B (Invitrogen), pET-11a (Stratagene) and pQE30 (Qiagen) plasmids with 6xhis tag, SacI, XhoI restriction enzyme sites. Fusion partners (trxA, nusA, malE and slyD) were amplified from E. coli genomic DNA and cloned into pBAD-dxs with NcoI and SacI sites. Erg12 was amplified from S. cerevisiae genomic DNA and cloned into the modified pBAD-B plasmid with 6xhis tag, SacI and Xhol restriction enzyme sites. Dxs mutants R398A [36], C32A, C330A, C457A and C32A-C330A-C457A were generated according the 'megaprimer' protocol [37]. Primers used in this study were summarized in Additional file 8 . All the pET-11a, pBAD-B and pQE30 based plasmids were transformed into $E$. coli BL21-Gold (DE3), $E$. coli $\mathrm{DH} 10 \mathrm{~B}$ and E. coli M15 respectively. pAC-LYC was co-transformed with all the plasmids except pBAD-erg12.

\section{E. coli growth and induction of protein expression}

A colony was picked from agar plate, inoculated into 2xPY medium (20g/L Peptone, 10g/L Yeast extract, and $10 \mathrm{~g} / \mathrm{L} \mathrm{NaCl}, \mathrm{pH}=7$ ) containing proper antibiotics, and incubated overnight. Ten microliter aliquots of overnight grown cell culture were inoculated into $1 \mathrm{~mL} 2 \mathrm{xPY}$ medium in $14 \mathrm{~mL}$ Falcon tube. Cells were grown at $37^{\circ} \mathrm{C} /$ $300 \mathrm{rpm}$ till OD595 reached the range of 0.5 1.0. The cells were then induced with $1 \mathrm{mM}$ IPTG (E. coli BL21-Gold (DE3) and E. coli M15) or $10 \mathrm{mM}$ L-arabinose (E. coli $\mathrm{DH} 10 \mathrm{~B})$ and grown at $37^{\circ} \mathrm{C}$ or $20^{\circ} \mathrm{C}$ for indicated time before collected for protein solubility assay or metabolite assay. Additives (sorbitol, betaine, phosphate, HEPES, mevalonate etc.) were also fed to cell culture upon induction if necessary.

\section{Prediction and quantification of protein solubility}

The revised WH algorithm [18] was used for prediction of protein solubility. Protein solubility was experimentally quantified by centrifugation [21] as described below. At $24 \mathrm{~h}$ after induction, cell suspension equivalent to $1 \mathrm{~mL}$ 
OD595=1.0 cells, was centrifuged, and the pellet was resuspended in 100uL B-PERII reagent (Pierce). The mixtures were vortexed at room temperature for $10 \mathrm{~min}$, and centrifuged at $16,000 \mathrm{~g}$ for $10 \mathrm{~min}$. The supernatant containing soluble cell lysates, and the pellets (resuspended in $100 \mathrm{uL} 2 \% \mathrm{w} / \mathrm{v}$ SDS) containing insoluble cell lysates were analyzed by SDS-PAGE. The SDS-PAGE gel was visualized by staining with instant blue (Gentaur), and image of the gel was processed and quantified by the software Quantity One (Bio-Rad). Protein solubility was defined as the quantity of the target protein in soluble cell lysates over that in total cell lysates (soluble cell lysates + insoluble cell lysates). Because ERG12 protein cannot be separated from an abundant endogenous protein on SDS-PAGE, it was detected by western blot analysis with anti-6xhis tag antibody (Penta-his Ab, Qiagen).

\section{In vitro quantification of dxs activity}

The DH10B strain - araBAD promoter system was used to produce DXS at $20^{\circ} \mathrm{C}$, whose catalytic activity was characterized in vitro. At $24 \mathrm{~h}$ after induction, insoluble cell lysates were prepared as described above except that they were well resuspended in $100 \mathrm{uL}$ NPI-10 $(50 \mathrm{mM}$ $\mathrm{NaH}_{2} \mathrm{PO}_{4}, 300 \mathrm{mM} \mathrm{NaCl}, 10 \mathrm{mM}$ imidazole, $\mathrm{pH}=8$ ) instead of $2 \% \mathrm{w} / \mathrm{v}$ SDS. $1 \mathrm{uL}$ the mixture was then incubated in $20 \mathrm{uL}$ in vitro reaction solution containing $40 \mathrm{mM}$ Tris $(\mathrm{pH}=6), \quad 10 \mathrm{mM}$ pyruvate, $20 \mathrm{mM}$ DL-glyceraldehyde 3-phosphate, $1 \mathrm{mM}$ thiamine diphosphate, $12.5 \mathrm{mM} \mathrm{MgCl}_{2}$ and $5 \mathrm{mM} \beta$-mercaptoethanol. The reaction was terminated by $1 \mathrm{~mL}$ acidic extraction solution (acetonitrile/ methanol/water 40:40:20, 100mM formic acid) after $2 \mathrm{~h}$ incubation at $37^{\circ} \mathrm{C}$, and formation of DXP was quantified by SPE UPLC-MS.

\section{SPE UPLC-MS quantification of DXP and MVAP}

Concentration of DXP and MVAP in cell culture was quantified by SPE UPLC-MS. At $5 \mathrm{~h}$ after induction, $50 \mathrm{uL}$ cell suspension was sampled and diluted in $1 \mathrm{~mL}$ acidic extraction solution (acetonitrile/methanol/water 40:40:20, $100 \mathrm{mM}$ formic acid) and centrifuged at $16,000 \mathrm{~g}$ for $1 \mathrm{~min}$. Supernatant was loaded to a cartridge holding $11 \mathrm{mg}$ LC$\mathrm{NH} 2$ resin (Sigma) that was activated by $200 \mathrm{uL}$ acidic extraction solution. The cartridge was centrifuged at 2,800g for $1 \mathrm{~min}$, and eluted with $100 \mathrm{uL} 1 \% \mathrm{w} / \mathrm{v} \mathrm{NH} 4 \mathrm{OH}$ that was subsequently neutralized by $0.75 \mathrm{uL}$ acetic acid. The eluate was analyzed by UPLC (Waters ACQUITY UPLC) - MS (Bruker micrOTOF II) as described below. Aqueous solution (A) containing $15 \mathrm{mM}$ acetic acid and $10 \mathrm{mM}$ tributylamine and methanol (B) were used as mobile phase with a UPLC C18 column (Waters CSH C18 $1.7 \mu \mathrm{m} 2.1 \mathrm{x} 50 \mathrm{~mm})$. The elution was done at $0.15 \mathrm{~mL} /$ min with gradient (start: $100 \% \mathrm{~A}, 1.8 \mathrm{~min}: 100 \% \mathrm{~A}$, 3.1min: 60\% A, 4.9min: 60\% A, 5.4min: 10\% A, 9.5min: 10\% A, 10min: 100\% A). Electrospray ionization was used and (TOF)MS was operated to scan $50-800 \mathrm{~m} / \mathrm{z}$ in negative mode with $-500 \mathrm{~V}$ end plate voltage and $4500 \mathrm{~V}$ capillary voltage. Nebulizer gas was provided in 1bar, drying gas temperature was $9 \mathrm{~mL} / \mathrm{min}$, and dry gas temperature was $200^{\circ} \mathrm{C}$. Sample injection volume was $5 \mu \mathrm{L}$. A range of $\mathrm{m} / \mathrm{z}$ was extracted from the acquired data for DXP (213.0170 \pm 0.03 , eluted at $5.6 \mathrm{~min})$ or MVAP (227.0315 \pm 0.03 , eluted at $6.7 \mathrm{~min})$. The integrated area of signal peak at its retention time then was calculated for the metabolites with the software provided by the manufacturer. Based on the integrated area of signal, concentration of DXP and MVAP were determined by interpolating from a standard dilution of the intermediates prepared in biological matrix.

\section{Additional files}

Additional file 1: Validation of the protein solubility quantification
method.
Additional file 2: Effects of sorbitol on solubility of DXR, ISPD, ISPE,
ISPF, ISPG, ISPH, IDI and ISPA.
Additional file 3: Expression of nonfunctional DXS R398A in E. coli
DH10B.
Additional file 4: Effects of sorbitol addition on production of MEP
and MEC.
Additional file 5: Effects of fusion partners on DXS solubility.
Additional file 6: Attempts at improving solubility of DXS by
protein mutagenesis.
Additional file 7: Addition of 500mM sorbitol did not improve
lycopene yield.
Additional file 8: Bacteria strains, plasmids and primers used in this
study.

Competing interests

The authors declare that they have no competing interests.

Authors' contributions

KZ and HPT conceived the study. KZ and RYZ carried out the experiments. GS and HPT supervised the study. KZ and HPT wrote the manuscript. All authors read and approved the final manuscript.

\section{Acknowledgments}

Financial support from Singapore-MIT Alliance (Flagship Research Project) is acknowledged.

\section{Author details}

${ }^{1}$ Chemical and Pharmaceutical Engineering, Singapore-MIT Alliance, 4 Engineering Drive 3, Singapore, Singapore. ${ }^{2}$ Department of Biochemistry Yong Loo Lin School of Medicine, National University of Singapore, 8 Medical Drive Blk MD7 \#05-04, Singapore 117597, Singapore. ${ }^{3}$ Department of Chemical Engineering, Massachusetts Institute of Technology,

77 Massachusetts Avenue, Cambridge, USA.

Received: 19 June 2012 Accepted: 7 November 2012

Published: 14 November 2012

\section{References}

1. Ro DK, Paradise EM, Ouellet M, Fisher KJ, Newman KL, Ndungu JM, Ho KA, Eachus RA, Ham TS, Kirby J, et al: Production of the antimalarial drug precursor artemisinic acid in engineered yeast. Nature 2006, 440(7086):940-943

2. Ajikumar PK, Xiao WH, Tyo KE, Wang Y, Simeon F, Leonard E, Mucha O, Phon TH, Pfeifer B, Stephanopoulos G: Isoprenoid pathway optimization 
for Taxol precursor overproduction in Escherichia coli. Science (New York NY 2010, 330(6000):70-74.

3. Hunter WN: The non-mevalonate pathway of isoprenoid precursor biosynthesis. J Biol Chem 2007, 282(30):21573-21577.

4. Ajikumar PK, Tyo K, Carlsen S, Mucha O, Phon TH, Stephanopoulos G: Terpenoids: opportunities for biosynthesis of natural product drugs using engineered microorganisms. Mol Pharm 2008, 5(2):167-190.

5. Westfall PJ, Pitera DJ, Lenihan JR, Eng D, Woolard FX, Regentin R, Horning T, Tsuruta H, Melis DJ, Owens A, et al: Production of amorphadiene in yeast, and its conversion to dihydroartemisinic acid, precursor to the antimalarial agent artemisinin. Proc Natl Acad Sci USA 2012, 109(3):E111-E118.

6. Kim SW, Keasling JD: Metabolic engineering of the nonmevalonate isopentenyl diphosphate synthesis pathway in Escherichia coli enhances lycopene production. Biotechnol Bioeng 2001, 72(4):408-415.

7. Alper H, Jin YS, Moxley JF, Stephanopoulos G: Identifying gene targets for the metabolic engineering of lycopene biosynthesis in Escherichia coli. Metab Eng 2005, 7(3):155-164.

8. Kang MJ, Lee YM, Yoon SH, Kim JH, Ock SW, Jung KH, Shin YC, Keasling JD, Kim SW: Identification of genes affecting lycopene accumulation in Escherichia coli using a shot-gun method. Biotechnol Bioeng 2005, 91(5):636-642

9. Yuan LZ, Rouviere PE, Larossa RA, Suh W: Chromosomal promoter replacement of the isoprenoid pathway for enhancing carotenoid production in E. coli. Metab Eng 2006, 8(1):79-90.

10. Jin YS, Stephanopoulos G: Multi-dimensional gene target search for improving lycopene biosynthesis in Escherichia coli. Metab Eng 2007, 9(4):337-347.

11. Wang C, Oh MK, Liao JC: Directed evolution of metabolically engineered Escherichia coli for carotenoid production. Biotechnol Prog 2000, 16(6):922-926

12. Lee PC, Mijts BN, Schmidt-Dannert C: Investigation of factors influencing production of the monocyclic carotenoid torulene in metabolically engineered Escherichia coli. Appl Microbiol Biotechnol 2004, 65(5):538-546.

13. Martin VJ, Pitera DJ, Withers ST, Newman JD, Keasling JD: Engineering a mevalonate pathway in Escherichia coli for production of terpenoids. Nat Biotechnol 2003, 21(7):796-802.

14. Chang MC, Eachus RA, Trieu W, Ro DK, Keasling JD: Engineering Escherichia coli for production of functionalized terpenoids using plant P450s. Nat Chem Biol 2007, 3(5):274-277.

15. Harker M, Bramley PM: Expression of prokaryotic 1-deoxy-D-xylulose-5phosphatases in Escherichia coli increases carotenoid and ubiquinone biosynthesis. FEBS Lett 1999, 448(1):115-119.

16. Lee KK, Jang CS, Yoon JY, Kim SY, Kim TH, Ryu KH, Kim W: Abnormal cell division caused by inclusion bodies in $\mathrm{E}$. coli; increased resistance against external stress. Microbiol Res 2008, 163(4):394-402.

17. Wilkinson DL, Harrison RG: Predicting the solubility of recombinant proteins in Escherichia coli. Biotechnology (N Y) 1991, 9(5):443-448.

18. Davis GD, Elisee C, Newham DM, Harrison RG: New fusion protein systems designed to give soluble expression in Escherichia coli. Biotechnol Bioeng 1999, 65(4):382-388.

19. Agostini F, Vendruscolo M, Tartaglia GG: Sequence-based prediction of protein solubility. J Mol Biol 2012, 421(2-3):237-241.

20. Tartaglia GG, Pechmann S, Dobson CM, Vendruscolo M: A relationship between mRNA expression levels and protein solubility in E. coli. J Mol Biol 2009, 388(2):381-389.

21. Hirose $S$, Kawamura $Y$, Yokota K, Kuroita T, Natsume T, Komiya K, Tsutsumi T, Suwa Y, Isogai T, Goshima N, et al: Statistical analysis of features associated with protein expression/solubility in an in vivo Escherichia coli expression system and a wheat germ cell-free expression system. J Biochem 2011, 150(1):73-81.

22. Niwa T, Ying BW, Saito K, Jin W, Takada S, Ueda T, Taguchi H: Bimodal protein solubility distribution revealed by an aggregation analysis of the entire ensemble of Escherichia coli proteins. Proc Natl Acad Sci USA 2009, 106(11):4201-4206.

23. Rodriguez-Villalon A, Perez-Gil J, Rodriguez-Concepcion M: Carotenoid accumulation in bacteria with enhanced supply of isoprenoid precursors by upregulation of exogenous or endogenous pathways. J Biotechnol 2008, 135(1):78-84

24. Gonzalez-Montalban N, Garcia-Fruitos E, Villaverde A: Recombinant protein solubility - does more mean better? Nat Biotechnol 2007, 25(7):718-720.
25. Baneyx F, Mujacic M: Recombinant protein folding and misfolding in Escherichia coli. Nat Biotechnol 2004, 22(11):1399-1408.

26. Panavas T, Sanders C, Butt TR: SUMO fusion technology for enhanced protein production in prokaryotic and eukaryotic expression systems. Methods Mol Biol 2009, 497:303-317.

27. de Marco A: Protocol for preparing proteins with improved solubility by co-expressing with molecular chaperones in Escherichia coli. Nat Protoc 2007, 2(10):2632-2639.

28. Xie X, Pashkov I, Gao X, Guerrero JL, Yeates TO, Tang Y: Rational improvement of simvastatin synthase solubility in Escherichia coli leads to higher whole-cell biocatalytic activity. Biotechnol Bioeng 2009, 102(1):20-28.

29. Prasad S, Khadatare PB, Roy I: Effect of chemical chaperones in improving the solubility of recombinant proteins in escherichia coli. Appl Environ Microbiol 2011, 77(13):4603-4609.

30. Zhou S, Grabar TB, Shanmugam KT, Ingram LO: Betaine tripled the volumetric productivity of $\mathrm{D}(-)$-lactate by Escherichia coli strain SZ132 in mineral salts medium. Biotechnol Lett 2006, 28(9):671-676.

31. Kim CW, Han KS, Ryu KS, Kim BH, Kim KH, Choi SI, Seong BL: N-terminal domains of native multidomain proteins have the potential to assist de novo folding of their downstream domains in vivo by acting as solubility enhancers. Protein Sci 2007, 16(4):635-643.

32. Han KY, Song JA, Ahn KY, Park JS, Seo HS, Lee J: Solubilization of aggregation-prone heterologous proteins by covalent fusion of stress-responsive Escherichia coli protein, SlyD. Protein Eng Des Sel 2007, 20(11):543-549.

33. Kapust RB, Waugh DS: Escherichia coli maltose-binding protein is uncommonly effective at promoting the solubility of polypeptides to which it is fused. Protein Sci 1999, 8(8):1668-1674.

34. Kyratsous CA, Silverstein SJ, Delong CR, Panagiotidis CA: Chaperone-fusion expression plasmid vectors for improved solubility of recombinant proteins in Escherichia coli. Gene 2009, 440(1-2):9-15.

35. Tsao CY, Wang L, Hashimoto Y, Yi H, March JC, Delisa MP, Wood TK, Valdes $J$ J, Bentley WE: LuxS coexpression enhances yields of recombinant proteins in escherichia coli in part through posttranscriptional control of GroEL. App/ Environ Microbiol 2011, 77(6):2141-2152.

36. Xiang $S$, Usunow $G$, Lange $G$, Busch $M$, Tong L: Crystal structure of 1-deoxy-D-xylulose 5-phosphate synthase, a crucial enzyme for isoprenoids biosynthesis. J Biol Chem 2007, 282(4):2676-2682.

37. Sarkar G, Sommer SS: The "megaprimer" method of site-directed mutagenesis. Biotechniques 1990, 8(4):404-407.

doi:10.1186/1475-2859-11-148

Cite this article as: Zhou et al:: Enhancing solubility of deoxyxylulose phosphate pathway enzymes for microbial isoprenoid production. Microbial Cell Factories 2012 11:148.

\section{Submit your next manuscript to BioMed Central and take full advantage of:}

- Convenient online submission

- Thorough peer review

- No space constraints or color figure charges

- Immediate publication on acceptance

- Inclusion in PubMed, CAS, Scopus and Google Scholar

- Research which is freely available for redistribution 